\title{
Quantum Monte Carlo Simulation for Atomic Resolution SEM/STEM Image
}

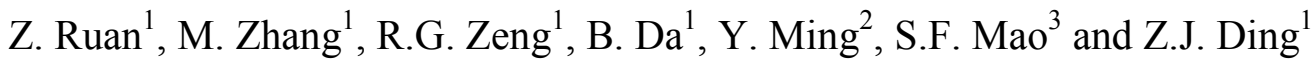 \\ 1. Hefei National Laboratory for Physical Sciences at Microscale and Department of Physics, University \\ of Science and Technology of China, 96 Jinzhai Road, Hefei, Anhui 230026, P.R. China \\ 2. School of Physics and Material Science, Anhui University, Hefei, Anhui 230601, P.R. China \\ 3. School of Nuclear Science and Technology, University of Science and Technology of China, 96 \\ Jinzhai Road, Hefei, Anhui 230026, P.R. China
}

Direct imaging of individual atoms with various electron signals has always been the most important challenge in electron microscopy. It has been reported only recently that an atomic resolution secondary electron (SE) image can be achieved in an aberration corrected scanning transmission electron microscope (STEM) by Zhu et al. [1] in 2009. The ability of simultaneous imaging at atomic scale of the specimen surface and of the sample bulk can have important applications in many research fields related to physics, materials and life science.

SE imaging is the most popular mode in a scanning electron microscope (SEM) and is widely used for its sensitivity to surface morphology. It has attracted many interests since the atomic resolution SE imaging for single atoms in a crystal was achieved by using a $200-\mathrm{keV}$ sub-angstrom-sized electron probe with aberration correction. Theoretical efforts have then been put into the exploration of mechanism for atomic scale SE imaging. However, it still lacks a complete simulation model which includes every physical factor in SE generation, transportation and emission in a crystalline solid to describe the real SE imaging process. In this work we have developed a new theoretical method to calculate atomic resolution SE image; the method combines Bohmian quantum trajectory method [2] for treating elastic scattering and diffraction for high energy incident electrons in a crystalline solid with a conventional Monte Carlo sampling of inelastic scattering events along quantum trajectory path. In this way the modification of classical electron trajectory by quantum mechanical trajectory in a crystal has enabled us to establish a quantum Monte Carlo method.

In our quantum Monte Carlo method, the simulation of atomic resolution SE imaging is made up of two steps. The first step concerns with high energy SE excitation in inner-shell ionization events as result of interaction of incident electrons with crystalline atoms. The interaction process involving diffraction of incident electrons is treated by the newly developed quantum Monte Carlo method. The second step deals with the cascade production and emission of low energy SE signals; this process is studied with a conventional Monte Carlo method [3] for an amorphous solid, which has been proven a powerful tool for study of SE signal.

High energy knock-on SEs generated in inner-shell ionization is simulated by the hydrogenic model [4]. Fig. 1(a) shows the calculated generalized oscillator strength (GOS) for transitions into the continuum from the $L_{23}$-shell of Si. The total ionization cross section of $\mathrm{Cu}$ for $K$-shell and $L$-shell obtained by the hydrogenic model is compared with Casnati ionization cross section in Fig. 1(b). With the hydrogenic model the impact parameter dependent inner-shell cross section is obtained and used for sampling of ionization events for an electron trajectory passing by an atom. The quantum Monte Carlo simulation is performed for a $\mathrm{Cu}$ fcc crystal in a lattice constant of $3.6 \AA$ and for the following microscope parameters: an aperture of $18 \mathrm{mrad}$, an accelerating voltage of $100 \mathrm{kV}$, a spherical aberration coefficient of $1.0 \mathrm{~mm}$, and a defocus of $-50 \mathrm{~nm}$. The simulated SE image is shown in Fig. 2(a), and the line profile 
of the SE image is given in Fig. 2(b). The atom columns and the double-layer structure of $\mathrm{Cu}$ crystal in fcc phase can be easily observed from the simulated SE image.

Our simulation clearly shows that, the high energy SE electrons generated in atomic inner-shell ionization by electron trajectories travelling along atom columns should be the first source of signals; while the further cascade multiplication of SE generation process has not destroyed fully the original local information on signal intensity. Simulation has thus led to a good understanding of the underlying physical mechanism of atomic resolution SE image.

\section{References:}

[1] Y. Zhu et al, Nat. Mater. 8 (2009), p. 808.

[2] R. G. Zeng and Z.J. Ding, J. Surf. Anal. 17 (2011), p. 198.

[3] Z. J. Ding and R. Shiimzu, Scanning 18 (1996), p. 92.

[4] R. F. Egerton, in "Electron Energy-Loss Spectroscopy in the Electron Microscope", (Springer US, New York) p. 184.

[5] The authors acknowledge funding from the National Natural Science Foundation of China (Nos. 11274288 and 11204289), the National Basic Research Program of China (Nos. 2011CB932801 and 2012CB933702), Ministry of Education of China (No. 20123402110034) and “111” project (No. B07033), and Chinese Academy of Sciences (No. XXH12503-02-02-07).
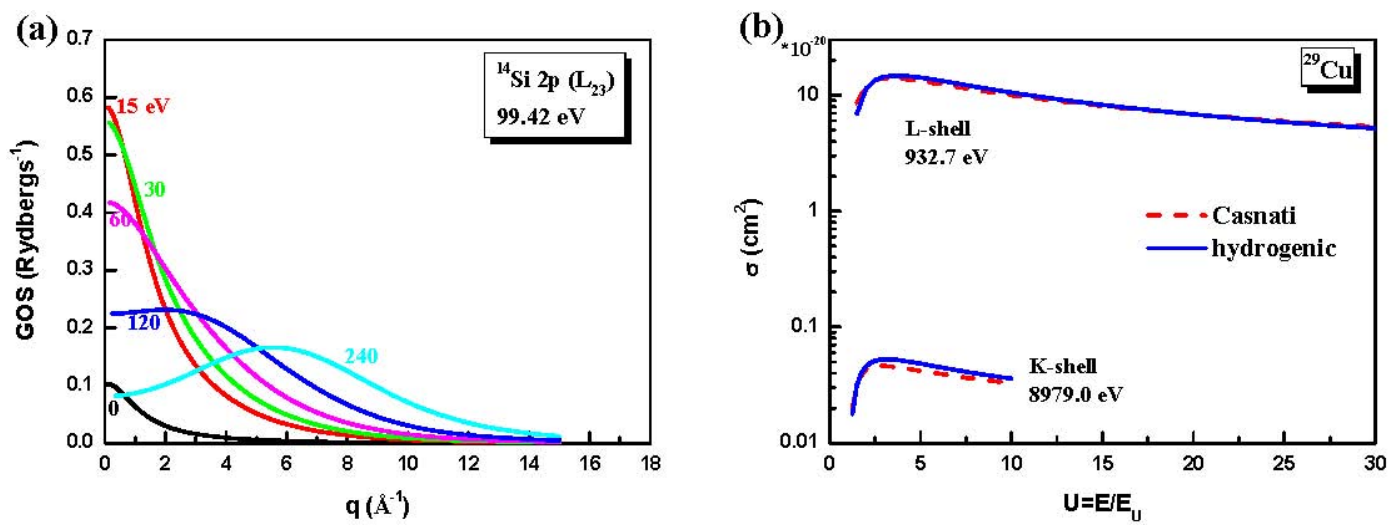

Figure 1. (a) GOS for transitions into the continuum from the $\mathrm{L}_{23}$-shell of Si. (b) The total ionization cross section of $\mathrm{Cu}$ for $\mathrm{K}-l$ and L-shells. The hydrogenic model is compared with Casnati cross section.

(a)

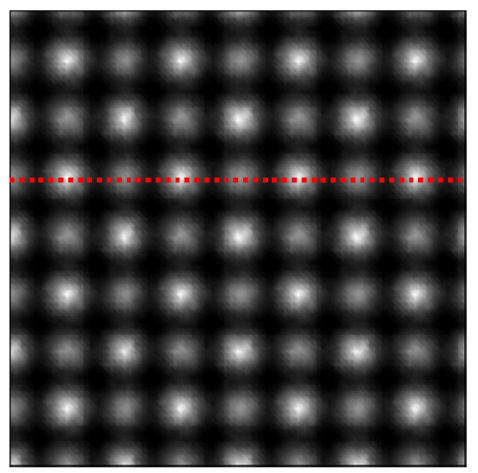

(b)

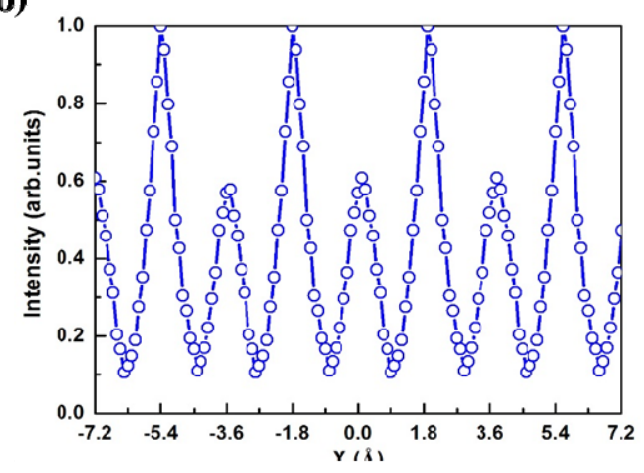

Figure 2. (a) Atomic resolution SE image simulated by the quantum Monte Carlo method. (b) Line profile along the dotted line in (a). 